Arts

et Savoirs

\section{Arts et Savoirs}

$2 \mid 2012$

Les théories de l'énonciation : Benveniste après un demi-siècle

\title{
Du potentiel sémantique au signifié dialogique
}

Réflexions sur la construction énonciative du sens

\section{Francesco La Mantia}

\section{(2) OpenEdition}

\section{Journals}

Édition électronique

URL : http://journals.openedition.org/aes/482

DOI : $10.4000 /$ aes.482

ISSN : 2258-093X

Éditeur

Laboratoire LISAA

Référence électronique

Francesco La Mantia, « Du potentiel sémantique au signifié dialogique », Arts et Savoirs [En ligne],

2 | 2012, mis en ligne le 15 juillet 2012, consulté le 03 mai 2019. URL : http://journals.openedition.org/ aes/482 ; DOI : 10.4000/aes.482

Ce document a été généré automatiquement le 3 mai 2019.

Centre de recherche LISAA (Littératures SAvoirs et Arts) 


\section{Du potentiel sémantique au signifié dialogique}

Réflexions sur la construction énonciative du sens

Francesco La Mantia

\section{Questions générales}

1 Dans cet article, je voudrais examiner la notion de potentiel sémantique et les rapports entre cette notion et ce que l'on appelle communément activité humaine de langage. En particulier, je voudrais poser ces deux questions : a) est-il est possible de parler d'une seule et même notion de " potentiel sémantique »? et b) l'activité humaine de langage est-elle indissociable de ces potentiels-mêmes, autrement dit, est-elle réductible ou irréductible à tout un ensemble d'opérations logico-cognitives qui portent nécessairement ou non sur ces potentiels-mêmes. Ma réponse à la première question est: non! Ma réponse à la deuxième question est : oui et non.

2 Afin de prouver le contenu de ces réponses, je voudrais préciser tout d'abord le statut épistémologique de la notion de potentiel sémantique et montrer, dans un deuxième temps, les raisons pour lesquelles, à mon avis, l'activité humaine de langage est à la fois réductible et irréductible à ces potentiels mêmes.

\section{Différentes représentations du potentiel sémantique}

Lorsqu'on se penche sur la notion de potentiel sémantique, il est nécessaire de distinguer deux représentations différentes de ce potentiel, c'est-à-dire le potentiel sémantique envisagé comme noyau de sens ancré au mot et le potentiel sémantique envisagé comme totalité des usages, des connaissances et des concepts impliqués par les emplois effectifs de ces mots.

4 La première représentation est susceptible, à son tour, d'être partagées en deux sousreprésentations successives, c'est-à-dire le potentiel sémantique envisagé comme signifié 
général des expressions-type, ou, en suivant la terminologie scientifique des philosophes du langage anglophones, le potentiel sémantique envisagé comme core-meaning invariant, et le potentiel sémantique envisagé comme forme schématique.

\section{Core-meaning}

5 Concernant la première sous-représentation, j'ai beaucoup de doutes à ce propos parce que le statut épistémologique du core-meaning n'est pas tout à fait clair. Qu'est-ce que le core-meaning d'un mot? S'agit-il d'un sens prototypique, d'un sens basique ou encore d'un sens littéral ? Bien entendu, en proposant ces différentes versions de core-meaning, je ne veux pas vraiment suggérer des solutions de rechange, mais, tout au contraire, montrer la nature hautement problématique et mystérieuse du core-meaning. Bref, qu'il soit envisagé comme sens prototypique, sens basique ou sens littéral, le core-meaning laisse tout de même trop de questions ouvertes.

6 En premier lieu, les rapports entre le core-meaning et les autres acceptions d'un mot ne sont pas évidents à appréhender; en deuxième lieu, il reste à montrer dans quel sens et dans quelle mesure le core-meaning est un noyau invariant. Ma perplexité sur le premier point est due au fait que, dans beaucoup d'approches différentes, ces rapports sont envisagés comme des affinités de contenu réglées par une sorte de couche de sens autonome qui cache, ou bien réduit à une unité, les multiplicités des emplois de chaque mot.

7 Par exemple, dans les sémantiques classiques du prototype, cet aspect «unifiant»du core-meaning est bien évident : on va repérer dans l'espace sémantique de chaque mot un sens central arbitraire et l'on décrit ce sens comme un lieu d'intersection au sein duquel toutes les conditions d'application du mot se croisent. Toutefois, ce point de vue est intenable car - comme l'ont bien montré les travaux en sémantique interprétative de Rastier (2003) - une représentation intersective, ou bien prototypique, des espaces sémantiques se heurte à beaucoup de difficultés irrésolubles : a) en premier lieu, c'est la notion même de sens central qui fait problème car les critères définitoires de centralité sémantique varient beaucoup selon les auteurs; b) en deuxième lieu, et au-delà de ces problèmes définitoires, ce sont les espaces sémantiques mêmes des mots qui de façon systématique rejettent la hiérarchie entre un centre et une périphérie. En fait, il est très fréquent de trouver de polysèmes qui ont plusieurs sens dont aucun ne domine clairement les autres; enfin, il est bien difficile de rendre compte de tous les emplois des mots par une seule et même signification.

8 Ma perplexité sur le deuxième point est due au fait qu'il n'y a pas de sens en dehors de celui qui est créé dans et par l'action commune de multiples facteurs co-textuels et contextuels. Donc, s'il y a construction du sens, chaque représentation du sens lexical comme core-meaning invariant est un construit théorique emprunté au laboratoire conceptuel du linguiste qui ne correspond pas aux pratiques langagières effectives des énonciateurs. En fait, l'idée qu'il soit possible de repérer un core-meaning invariant est étroitement liée au préjugé selon lequel on peut toujours isoler chaque mot du tissu des facteurs co-textuels et contextuels qui l'entourent.

9 Toutefois, il est facile de montrer que cette représentation du sens lexical ne tient pas à l'épreuve des faits. Il faut observer que chaque mot isolé n'est pas vraiment isolé, car il est toujours inséré dans un contexte de régularité spécifique, c'est-à-dire l'espace logique du dictionnaire - et c'est dans cet espace particulier qu'il est envisagé comme tel. En 
outre, il ne faut pas confondre une chose avec une autre : l'espace logique du dictionnaire ne constitue pas un bon modèle de fonctionnement du sens, car il ne représente qu'une partie infinitésimale des intuitions du locuteur. D'où l'on déduit l'inadéquation profonde de la représentation initiale : le core-meaning - loin d'être un noyau de sens invariant - est lui-même le résultat d'interactions co-textuelles qui se constituent au niveau de particulières coordonnées énonciatives. Il nous reste donc à examiner la deuxième sousreprésentation, c'est-à-dire le potentiel sémantique envisagé comme forme schématique.

\section{Forme schématique}

10 Je sais qu'il s'agit bien d'une notion très connue parmi les linguistes qui se sont formés avec la T.O.P.E d'Antoine Culioli, mais je sais aussi bien que cette notion a soulevé plusieurs difficultés d'interprétation chez les culioliens et, plus en général, beaucoup de doutes chez les chercheurs qui ont réfléchi de façon systématique sur les phénomènes de polysémie.

11 Il est fort probable que ces difficultés et ces doutes soient principalement dus à l'ambivalence constitutive de cette notion et au fait qu'elle ne résout qu'une partie infinitésimale des problèmes concernant ces phénomènes-mêmes - laissant, tout au contraire, trop des questions ouvertes. Pour ma part, j'en examinerai que celle qui concerne les rapports entre formes schématiques et diversités des emplois des unités qu'elles caractérisent - ou bien qu'elles devraient caractériser.

12 La littérature d'obédience culiolienne est sur ce point très claire: on peut décrire le sémantisme de chaque mot par une forme schématique associée qui en modélise les divers emplois en co-texte (et/ou en contexte). Chaque forme est, sous cet aspect particulier, un noyau de sens abstrait qui ne correspond pas en lui-même à aucune valeur particulière de l'unité examinée mais qui est au même temps compatible avec les différentes valeurs de cette unité. On pourrait dire que chaque forme schématique est un "pôle d'invariance", ou bien un "pôle régulateur", qui doit rendre compte des variations sémantiques réglées des unités linguistiques. D'autre part, elle est caractérisée comme un noyau de sens qui est à la fois instable et déformable. Ces propriétés de déformabilité et instabilité - tout à fait absentes des sémantiques cognitives à base prototypique - rendent la forme schématique de chaque marqueur sensibles aux interactions entre ce marqueur-même et ses éventuels voisinages co-textuels.

Comme les culioliens nous le répètent, ces interactions co-textuelles assument l'aspect de rapports de détermination réciproque entre la forme schématique du marqueur et ses indices co-textuels - et c'est en vertu de ces rapports qu'il y a construction du sens. En fait, au cours de telles interactions, la forme schématique, qui est susceptible d'être remaniée par l'action de détermination réciproque entre cotexte et marqueur, opère comme un générateur potentiel de sens. Bref : au cours de telles interactions, chaque forme se déforme et, en se déformant de manière continue, donne lieu à l'extrême variété des effets de sens possibles.

D'où l'ambivalence constitutive de cette notion: d'un côté, elle est un invariant; de l'autre, elle est - en suivant la terminologie de Stéphane Robert (2006) - une « matrice de sens " ${ }^{1}$ à la fois instable et déformable. En soi telle ambivalence ne fait pas problème. Mais beaucoup de problèmes surgissent lorsque l'on en examine un aspect particulier. Cet aspect, qui concerne l'invariance de la forme schématique, peut être ramené à un principe de définition - connu sous le nom de principe d'extensionalité - selon lequel « la 
forme schématique doit être compatible avec tous les emplois du marqueur qu'elle décrit. $\aleph^{2}$ Tel principe, examiné sous la perspective d'une linguistique historique, détermine une tension irrésolue entre l'invariance de la forme schématique et le fait que les emplois du marqueur varient (ou peuvent varier) dans le temps - et parfois de façon si imprévisible que la forme schématique ne peut plus en rendre compte.

Il a été, à juste titre, observé par Dufaye (2009) que si la forme schématique ne peut pas expliquer certains emplois, alors on peut supposer qu'elle est incomplète et/ou inadéquate ${ }^{3}$. Il s'agit sans doute d'une indication méthodologique précieuse, car elle impose une très grande prudence dans l'analyse de tel concept en montrant en outre qu'il n'est pas possible de parler de forme schématique avant d'avoir étudié tous les emplois du marqueur examiné. Mais le fait demeure que la tension dont on a déjà parlé reste ouverte, puisque les totalités des emplois par rapport auxquelles est évaluée la complétude d'une forme schématique donnée sont tout de même relatives à un corpus donné qui ne peut pas couvrir toutes les variations diachroniques dont est susceptible le marqueur. D'où une limite constitutive de cette approche, c'est-à-dire l'impossibilité de maintenir un lien continu à la diachronie.

\section{Contextes d'usage}

Par conséquent, l'exigence de rendre compte de tels phénomènes nous pousse vers la deuxième représentation du potentiel sémantique, c'est-à-dire le potentiel sémantique envisagé comme totalité des usages possibles des mots que l'on décrit. En fait, telle représentation nous permet de décrire le changement sémantique des items lexicaux non pas en termes de déformation de noyaux de sens ancrés aux unités (ou séquences d'unités) co-textuelles, mais plutôt en terme des variations en temps réel qui sont induites - ou suggérées - par le contexte d'usage. En vertu de ce déplacement d'attention vers le contexte d'usage, il est tout à fait possible de prendre en charge les aspects diachroniques du changement sémantique.

L'idée générale, qui domine dans une telle conception du potentiel sémantique et qui trouve sa place dans la théorie de l'inférence invitée de Traugott et Dasher (2002), est que l'identité de chaque marqueur est une identité fluide qui se définit en fonction de deux paramètres étroitement liés : a) l'histoire singulière du marqueur; b) les contextes d'insertion du marqueur - là où par contexte d'insertion on pourrait entendre un ensemble cohésif de relations distribuées entre facteurs hétérogènes de détermination du signifié : relations entre mots et co-texte, entre co-texte et contexte, entre énonciateurs et co-énonciateurs. De ce point de vue, chaque emploi du marqueur correspond à une mise en œuvre unique et singulière de tel ensemble cohésif qui constitue le véritable potentiel sémantique du marqueur - là où telle « mise en œuvre " réfléchirait à son tour les évolutions sémantiques du marqueur et les processus basés sur l'usage responsables de telles évolutions.

Bref, de tels processus se configurent comme des dispositifs sémantico-pragmatiques qui, d'un côté, activent en temps réel des nouvelles valeurs du marqueur - et ce sont ces valeurs que l'on appelle communément inférences invitées; de l'autre, ils sont en mesure d'en neutraliser certains et/ou d'en coder certains autres dans des contextes d'interprétation particuliers. 


\section{Conclusion}

19 D'où ma réponse à la deuxième question : l'activité langagière est à la fois réductible et irréductible à la notion de potentiel sémantique, car, d'un côté, on peut envisager tel potentiel comme un noyau de sens ancré aux mots, de l'autre, comme des relations distribuées entre facteurs hétérogènes de détermination du signifié qui mélangent connaissance des langues et connaissance du monde.

20 Si l'on adopte la première conception du potentiel sémantique, il est fort clair que l'activité langagière ne se réduit pas à des opérations qui portent sur ces potentielsmêmes, puisque ce qu'on appelle activité de langage n'est pas un acte isolé du monde, c'est-à-dire un ensemble d'opérations réductibles à la déformation des noyaux de sens pré-donnés avec chaque mot.

21 Mais si nous adoptons la deuxième conception du potentiel sémantique, alors il est fort évident que cette activité est réductible à ces opérations qui portent sur ces potentiels mêmes, puisqu'ils constituent de lieux d'articulation hybride qui sont toujours façonnés par les rapports dialogiques qui vont s'instituer en temps réel entre énonciateur et coénonciateur. J'en viens aux conclusions - et en venant aux conclusions, je vais vous dire tout de suite qu'il reste beaucoup de travail à faire et qu'il faudra régler ce travail même en suivant, du moins, trois directions différentes : 1 ) élaborer une théorie adéquate des potentiels sémantiques qui soit apte à rendre compte des phénomènes d'hybridations constants entre connaissance de la langue et connaissance du monde ; 2) éclaircir la grammaire de l'expression potentiel sémantique, ou si l'on préfère, construire une carte conceptuelle qui soit apte à décrire les sens différents de cette expression même ; 3) établir quels modèles formels peuvent nous donner une description plus fine des régimes d'organisations qui du potentiel sémantique portent à la constitution des régimes dialogiques entre énonciateur et co-énonciateur. Il s'agit là de questions lourdes en termes de conséquences épistémologiques, et qu'il faudra par conséquent chercher à approfondir dans le cadre d'une analyse théorique adéquate.

\section{BIBLIOGRAPHIE}

ALLWOOD, Jens, « Meaning potentials and context: Some consequences for the analysis of the variation of meaning ", in Hubert Cuyckens, René Dirven \& John Taylor (dir.), Cognitive Approaches to Lexical Semantics, Berlin, 2003, p. 29-65.

COL Gilles, APTEKMAN Jean, GIRAULT Stéphanie \& VICTORRI Bernard, Compositionnalité gestaltiste et construction du sens par instructions dynamiques, CogniTextes, Volume 5, 2010. Voir le site : http:// cognitextes.revues.org/372.

DUFAYE, Lionel, Théorie des opérations énonciatives et modélisation, Paris, Éditions Ophrys, 2009.

NORÉN, Kerstin \& LINNEL Per, « Meaning Potentials and the interaction between lexis and contexts : an empirical substantiation », in Pragmatics, 17 :3.387-416, 2007. 
RASTIER, François, Arti e scienze del testo. Per una semiotica delle culture, Roma : Édition Meltemi, 2003.

ROBERT, Stéphane, « Polygrammaticalisation, grammaire fractale et propriétés d'échelle », in Robert Stéphane (dir.), Perspectives synchroniques sur la grammaticalisation : Polysémie, transcatégorialité et échelles syntaxiques, Éditions Peteers Louvain, 2006, p. 85-120.

TRAUGOTT, Elizabeth \& DASHER, Richard B., Regularity in semantic change, Cambridge, Cambridge University press, [2002] 2005.

VICTORRI, Bernard, « Un modèle mathématique et informatique de la polysémie », in Cahiers de la Maison des Sciences Humaines de l'Université de Caen, $\mathrm{n}^{\circ}$ 1, « Cognition et Langage, Axe modélisation en Sciences Humaines ", édité par Jean Vivier, 1993.

\section{NOTES}

1. Robert (2006, p. 98)

2. Dufaye (2009, p. 138)

3. Dufaye (2009, p. 138)

INDEX

Mots-clés : sens, potentiel sémantique, langage, core meaning, forme schématique

\section{AUTEUR}

\section{FRANCESCO LA MANTIA}

Università di Palermo, Dipartimento Fieri-Aglaia (la_mantiafrancesco@hotmail.com) 